\title{
Elementos para un modelo de negocio en servicios especializados. El caso de roaming internacional
}

\begin{abstract}
RESUMEN
El roaming es un servicio de comunicaciones móviles donde los clientes de un switch, denominado red origen, pueden traficar en otra red distinta a la que se denomina red visitada. Es la capacidad de un cliente móvil para efectuar y recibir llamadas con su mismo número celular cuando viaja fuera del país.

Este artículo presenta los elementos para el desarrollo de infraestructura del servicio, considerando que representa una oportunidad de negocio.

Palabras clave: Roaming, oportunidad, estrategia infraestructura, modelo de negocio.

ELEMENTS FOR A BUSINESS MODEL IN SPECIALIZED SERVICES. THE CASE OF INTERNATIONAL ROAMING

\section{ABSTRACT}

Roaming is a service of mobile communications where the clients of a switch named home network, can use cellular service in another different network named visited network. It is the capacity of a mobile user to carry out and to receive calls with same cellular number when he travels another countries. This article presents the elements for the service infrastructure development, considering that represents an opportunity of business.
\end{abstract}

Key words: Roaming, opportunity, strategy, infrastructure, model of business.

\section{CÓMO SURGE EL SERVICIO}

\section{ANTECEDENTES DEL ROAMING}

El roaming se define como la capacidad del cliente móvil de efectuar y recibir llamadas con su mismo número y su mismo equipo cuando viaja al extranjero, utilizando temporalmente la red de otro operador. El roaming es un servicio interoperadores donde convergen los intereses de diversos actores: gobiernos, fabricantes, proveedores, mayoristas, operadores, operadores virtuales y usuarios.

El crecimiento del roaming se potencia con la globalización, paradójicamente, los usuarios efectivos son pocos y repetitivos, conformando un segmento de mercado inelástico al precio. Esta situación determina que el BID (Banco Interamericano de Desarrollo) lo incluya dentro de los proyectos de desarrollo de infraestructura (2008).

Los viajeros de los países de la región se dirigen a un grupo de países que representan sus principales intereses comerciales. El cuadro 1 muestra la estructura del destino roaming para cualquier operador de América Latina, esta composición ha de variar en el futuro cercano, a medida que se implementen los nuevos acuerdos comerciales que diversifican las fuentes de aprovisionamiento de bienes y servicios, acercando a los países hacia las economías de la región y al Asia-Pacífico.

Después de una década en América Latina, representa la oportunidad de negocio desaprovechada, con ingresos potenciales que los operadores de la región dejan de recibir. Su mercado natural son los viajeros internacionales, aunque su uso está restringido a los clientes pospago de alto perfil (denominados VIP: very important person) que configuran un nicho de rentabilidad marginal significativa, pero cuya magnitud y efecto en los resultados totales, no es relevante, lo que genera preocupación de los organismos internacionales como Citel-OEA (2008), la coordinación de institutos privados como Regulatel-Ahciet (2007), el estudio de la academia como la Universidad del Cauca (2003), el interés por el valor creado (Band 1991), y las gestiones de los representantes de los fabricantes de tecnología como CDG (2004) y GSM Association (2006), aunque con pobres resultados, en general.

1 Magíster en Administración. Profesor en la Facultad de Ingeniería Industrial, Departamento Académico de Producción y Gestión Industrial de la UNMSM. E-mail: aacevedo@speedy.com.pe 


\begin{tabular}{|c|c|c|c|}
\hline & $\begin{array}{l}\% \text { tráfico } \\
\text { roaming }\end{array}$ & $\begin{array}{l}\text { Tendencia } \\
\text { del trafico }\end{array}$ & $\begin{array}{c}\text { \% trafico al } \\
2010 \\
\text { (estimado) }\end{array}$ \\
\hline $\begin{array}{l}\text { Norteamérica } \\
\text { (USA Canadá } \\
\text { y México) }\end{array}$ & 65 & Aumento & 40 \\
\hline $\begin{array}{l}\text { Países limí- } \\
\text { trofes }\end{array}$ & 22 & Aumento & 30 \\
\hline $\begin{array}{l}\text { Europa occi- } \\
\text { dental* }\end{array}$ & 10 & Aumento & 20 \\
\hline $\begin{array}{l}\text { Otros países } \\
\text { de América } \\
\text { Latina }\end{array}$ & 3 & Estacionario & 5 \\
\hline $\begin{array}{l}\text { Tigres } \\
\text { asiáticos }\end{array}$ & 0 & aumento & 4 \\
\hline $\begin{array}{l}\text { Resto del } \\
\text { mundo }\end{array}$ & 0 & & 1 \\
\hline
\end{tabular}

* España, Francia, Italia

Fuente: Elaboración propia basado en estadísticas GSM/IFAST

Inicialmente, el operador que impulsó el roaming con Estados Unidos de Norteamérica (USA) fue AT\&T Wireless (AT\&TW), su buen servicio en tecnología TDMA permitía un eficaz servicio roaming, pero adolecía de alto riesgo de fraude.

La Code División Multiple Access (CDMA) es la tecnología digital de acceso múltiple por división de códigos, usado en las comunicaciones móviles según el estándar US (IS 95) en el intervalo de frecuencias 800-1.900 MHz, es la tecnología utilizada por los operadores americanos que emplea codificación avanzada para que varios usuarios compartan un mismo bloque del espectro de ancho de banda para trasmitir llamadas digitalizadas de voz y datos. La time division multiple access (TDMA) es la tecnología digital de acceso múltiple por división de tiempo, que separa la trasmisión de múltiples llamadas sobre un número finito de frecuencias, permitiendo la transmisión de varias conversaciones simultáneas. CDMA y TDMA son las tecnologías inalámbricas que se han disputado la primacía de su estándar tecnológico en telefonía celular y en PCS.

Cuando los operadores migraron a tecnología CDMA se manifestaron las casi insalvables restricciones regulatorias, tecnológicas, de mercado y estratégicas que retrasaron el funcionamiento adecuado en América. Mientras tanto, en Europa, se aplicaba la decisión política de utilizar el estándar tecnológico TDMA que se ha denominado GSM (Global System for Mobile communication) en la Co- munidad Europea, facilitando la integración de redes y eliminando las posibilidades de tecnologías no compatibles.

En la región, la estrategia ha sido diferenciada y no concordada entre los países y los operadores. En CDMA se propugna el libre desarrollo que ha llevado a tecnologías "propietarias" aparentemente compatibles, que ha impedido el crecimiento del servicio a niveles adecuados. En GSM, se ha creado diferencia competitiva a su favor, mediante la decisión de los países de la unión europea, de eliminar la libertad de elección tecnológica, lo que le permitió aventajar a la otra opción en pugna.

El servicio roaming se conforma con una arquitectura multidimensional, organizada bajo el enfoque de proceso para coordinar la actividad roaming a cargo de las áreas funcionales.

El proceso roaming comprende tres partes:

1) El segmento administrativo, se maneja bajo administración propia y representa los gastos administrativos por la coordinación multifuncional y multioperadores.

2) El segmento técnico, comprende una parte de inversión y administración propia y otra parte tercerizada bajo modalidad de cargos fijos o por volumen.

3) El terminal es el tercer segmento, que se transfiere al cliente y no se considera inversión.

A pesar del potencial de mercado del roaming, éste no crece y el fraude ha generado pérdidas en algunos operadores. Algunas claves para el retraso, se encuentran en la falencia de normatividad clara, la relación precio-calidad, pobre cooperación entre operadores, altas inversiones sin contrapartida de ingresos. Otros temas críticos son la interoperabilidad tecnológica y la interconexión de redes. Los temas estratégicos del roaming son el diagnóstico y las restricciones para el crecimiento, la definición del futuro modelo empresarial, la estrategia regional de desarrollo, la mejora de la calidad, la satisfacción del roamer, el control y seguridad para evitar fraude y, sobre todo, la magnitud de la infraestructura crítica requerida para un servicio adecuado.

\section{EL SERVICIO ROAMING EN AMÉRICA LATINA}

\section{El surgimiento del servicio}

El servicio roaming surge en los años 90 , a la par del crecimiento de las tecnologías analógica y TDMA. En Sudamérica aparece con los acuerdos 
bilaterales entre operadores, donde cada operador local brindaba un bloque de MIN (mobile identification number) que es el número de identificación personal que se asigna a cada terminal o handset a fin que el otro operador grabe el número en un segundo NAM (numeric assignment module) que es un circuito integrado donde se graba información del número de teléfono celular, vía fax se coordinaba la activación por un corto período de tiempo. Esto generó alto riesgo y elevado fraude por suscripción y clonaje de equipos.

El roaming con EE.UU. se inicia con el fuerte apoyo de AT\&TW, en tecnología TDMA y con elevado riesgo de fraude. El gateway inicial fue el propio AT\&TW y la facturación se realizaba a través de la empresa de software informático EDS (Electronic Data Systems), posteriormente se migró a un proveedor de servicios denominado GTE-TSI (GTE-Telecommunications Systems Incorporated) luego denominado Syniverse. Bellsouth Wireless implantó su propio gateway llamado Pan American Roaming Consortium (PARC) que manejaba servicios de interconexión, facturación y fraude entre los operadores de su alianza.

En Europa, se acordaba el desarrollo tecnológico unificado para los países de la Unión, mediante un único y común estándar tecnológico.

En los primeros años del nuevo siglo, los operadores profundizan su migración hacia la norma GSM, lo que arrincona a los grandes operadores CDMA
(Verizon Wireless, Sprint, Nextel). La decisión de Cingular Wireless (AT\&TW) de migrar su tecnología hacia GSM, fue rápidamente imitada por los operadores internacionales Telmex y Telefónica.

\section{La infraestructura para el servicio}

El roaming se sustenta en una red internacional conectada a través de gateways que operacionalizan la interconexión, la traducción de protocolos y la transmisión en línea del perfil del cliente, desde el switch origen hacia el switch visitado, a fin de período también realizan el intercambio de los registros de llamadas o cdr's (call data records) en formato de facturación.

Los principales gateways son los proveedores Syniverse y MACH (que ha absorbido el PARC de Bellsouth y el clearing financiero de Cibernet), ambos concentran casi el $100 \%$ del tráfico internacional. Estos gateways son los intermediarios que facilitan el servicio interstandar e inter-tecnologías, y prácticamente controlan el tráfico roaming a nivel mundial. Representan una elevada porción del costo de operación del servicio. Un caso especial es Brasil, cuyo tamaño de mercado determina el uso de un gateway que facilita el roaming entre los operadores establecidos en este país (ver la figura 1).

Existen algunos operadores que eluden el uso de gateways y los pagos por interconexión. Específicamente, un gran operador europeo utiliza un elaborado modelo basado en el elevado volumen de

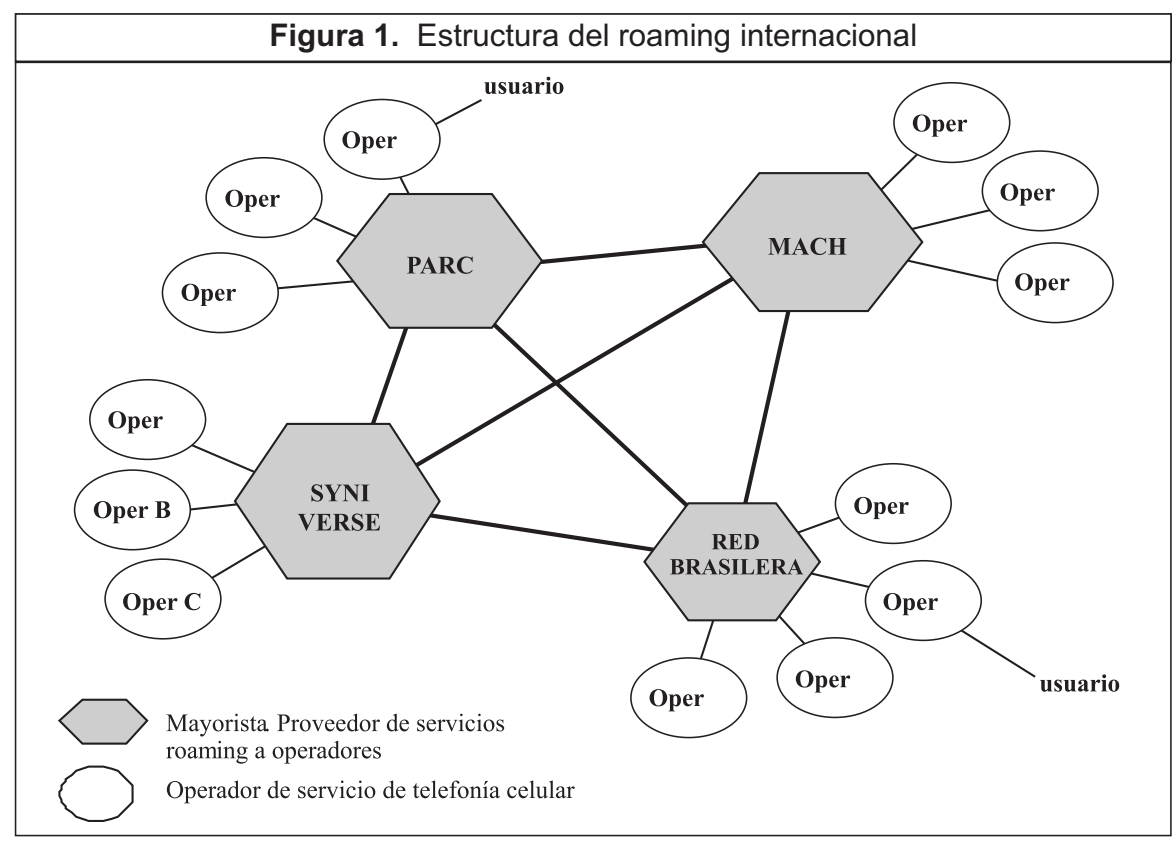

Fuente: Elaboración propia. 
viajeros entrantes a su país, las exigencias de otros operadores a su gateway y su negativa a pagar cualquier servicio de estos intermediarios. "Perro muerto" le llaman en Perú.

La dificultad mayor reside en el modelo de negocio aplicado para roaming, se sustenta en acceder a clientes VIP de alto consumo local y baja sensibilidad al precio, a los que se les carga un significativo markup para cubrir los costos y las contingencias imprevisibles (fraude en roaming, no facturación o no cobro, churn de clientes).

La inversión en infraestructura significa la consolidación de un servicio. El roaming se ha definido como un factor importante para la comunicación e integración de las alianzas empresariales, por lo que, su desarrollo está considerado prioritariamente dentro del paquete de proyectos de creación de infraestructura del BID (2008).

\section{EL PROCESO ROAMING}

\section{IDENTIFICACIÓN DE LA OPORTUNIDAD DE NEGOCIO}

\section{Estimación del tamaño de mercado}

El mercado de viajeros entrantes hacia América Latina, supera los 130 millones de viajes representando el $60 \%$ de la población total.

Bajo la premisa evidente de que la densidad celular tiende al $100 \%$ (toda la población posee un celular, en promedio), es factible afirmar que todo viajero internacional entrante es un usuario real y todo viajero internacional saliente es un usuario potencial de roaming.

Para definir el tamaño de mercado, presentamos un ejercicio sobre el target de roamers en un hipotético país A y América Latina, con datos estimados al 2007. El cuadro 2 presenta el potencial de roamers e ingresos potenciales, estos estimados se sustentan en un moderado ingreso por minuto de US\$ 0,50 y un costo de US\$ 0,20 por llamada.

El país A tendría posibilidades de ingresos anuales por roaming que superarían 32 millones de dólares, con margen neto de 24 millones (lo cual brinda una apreciable base para incrementar el servicio mediante estrategia de bajo precio).

El tamaño de mercado de servicios de roaming en América Latina podría llegar a los tres mil millones de ingresos anuales.
Cuadro 2. Potencial de mercado en roaming

\begin{tabular}{|l|c|c|}
\hline & PAIS A & $\begin{array}{c}\text { América } \\
\text { atina }\end{array}$ \\
\hline $\begin{array}{l}\text { Viajeros entrantes } \\
\text { del exterior }\end{array}$ & 1,500 & 131,955 \\
\hline $\begin{array}{l}\text { Viajeros salientes al } \\
\text { exterior (estimado) }\end{array}$ & 800 & 100,000 \\
\hline
\end{tabular}

\section{Potencial de ingresos roaming entrante}

\begin{tabular}{|l|c|c|}
\hline $\begin{array}{l}\text { Target: 30\% viajeros } \\
\text { entrantes }\end{array}$ & 500 & 43,985 \\
\hline $\begin{array}{l}\text { Ingreso \$ - min } \\
\text { totales }\end{array}$ & 22,500 & $1,979,325$ \\
\hline \multicolumn{2}{|c|}{ Potencial de ingresos roaming saliente } \\
\hline $\begin{array}{l}\text { Target: 20\% } \\
\text { viajeros salientes }\end{array}$ & 160 & 20,000 \\
\hline $\begin{array}{l}\text { Ingreso \$ - min } \\
\text { totales }\end{array}$ & 9,600 & $1,200,000$ \\
\hline $\begin{array}{l}\text { Ingreso \$ anual } \\
\text { potencial total }\end{array}$ & 32,100 & $3,179,325$ \\
\hline
\end{tabular}

Fuente: Acevedo, A. Calidad e interoperabilidad en el servicio roaming: claves para el modelo de negocio. En www.iirsa.org/BancoEvento/T/taller_iirsa_citel/.

\section{La estrategia que retrasa la consolidación del servicio}

El uso efectivo del roaming está circunscrito a un segmento de clientes de alto pago y baja elasticidad-precio. Su perfil elemental es: empresarios, ejecutivos y funcionarios que realizan entre tres y ocho viajes al exterior por año, el consumo es asumido por la organización a la que representan, su rentabilidad individual es elevada. Este segmento, denominado heavy-user tiene capacidad decisoria y alta participación en los negocios internacionales y en los procesos de integración (cuadro 3).

Los segmentos de clientes normales y los clientes eventuales presentan características que, de entenderse, podrían representar elevar los ingresos a niveles superiores a los 3,200 millones de dólares, que se han definido como el tamaño potencial de mercado roaming. Por el inadecuado enfoque de mercadeo no se capta un sector más amplio de usuarios, y se está dejando de percibir un gran volumen de ingresos potenciales. 


\begin{tabular}{|c|c|c|c|}
\hline \multirow[t]{2}{*}{ Variables } & \multicolumn{3}{|c|}{ Tipos de usuarios } \\
\hline & $\begin{array}{l}\text { Heavy } \\
\text { user }\end{array}$ & Mediano & $\begin{array}{l}\text { Esporá- } \\
\text { dico }\end{array}$ \\
\hline Viajes al año & 03-Ago & 01-Mar & $1 / 2-1 *$ \\
\hline Días por viaje & 3 & 3 & 5 \\
\hline $\begin{array}{l}\text { Llamadas por } \\
\text { viaje }\end{array}$ & 30 & 20 & 20 \\
\hline $\begin{array}{l}\text { Minutos por lla- } \\
\text { mada }\end{array}$ & 5 & 3 & 3 \\
\hline \multicolumn{4}{|l|}{$\begin{array}{l}\text { Composición de } \\
\text { tráfico }\end{array}$} \\
\hline saliente & $80 \%$ & $80 \%$ & $90 \%$ \\
\hline entrante & $20 \%$ & $20 \%$ & $10 \%$ \\
\hline \multicolumn{4}{|l|}{$\begin{array}{l}\text { Curva ABC (solo } \\
\text { usuarios roa- } \\
\text { ming) }\end{array}$} \\
\hline$\%$ de usuarios & $2 \%$ & $8 \%$ & $90 \%$ \\
\hline$\%$ de ingresos & $95 \%$ & $4 \%$ & $1 \%$ \\
\hline
\end{tabular}

* $1 / 2$ viaje: un viaje cada dos años

Fuente: Elaboración propia basado en estadísticas GSM/IFAST

Se ha iniciado una estrategia de reemplazo de la tecnología CDMA (dominante en USA), por la tecnología GSM (dominante en Europa) debido al bajísimo costo de inversión por punto de usuario, por la eficiencia en los upgrades (tiempo de espera y costo), la facilidad para interconectar redes y la oferta de un roaming estandarizado.

Estas diferencias de GSM no generan ventajas competitivas, ya que los directivos locales (designados por la Dirección, desde un país europeo o Norteamérica) no han invertido ni creado infraestructura para sostener el servicio, debido a su, tal vez, formación humanística, escasa formación o ínfimo entendimiento de la concepción sistémica del negocio.

Ha surgido la paradoja del cambio, donde se migra a una tecnología conveniente, pero no se aprovechan sus diferencias competitivas, referidas a un roaming sencillo y de bajo costo interoperadores.

\section{Los servicios requeridos}

Desde la perspectiva del cliente, el roaming internacional, en el corto y mediano plazo, se ha de concentrar en los servicios de voz:
- Para la presente década, la comunicación de voz seguirá representando el producto celular más importante.

- Los servicios de voz, representan y representarán el servicio vaca lechera (generador de ingresos) y el mayor mayor volumen de ingresos (más del $80 \%$ ), sustentará la inversión en nuevos desarrollos y la búsqueda de la killer application (se define así a la aplicación comercial definitiva, es el producto ganador que sustentará el servicio).

- Los servicios de voz y los agregados de voz, son los determinantes de la satisfacción del cliente y la rentabilidad del negocio.

- Los servicios de datos han de representar la oferta para clientes sofisticados, de altas prestaciones y bajo pago.

\section{ELEMENTOS DEL MODELO DE NEGOCIO}

\section{La estrategia roaming en la región}

La visión del roaming y las estrategias de los operadores se diferencian por países y regiones. Los operadores de Estados Unidos y mexicanos han desarrollado un servicio basado en gateways privados que interconectan a los operadores de diferentes países. El costo de intermediación es alto, la calidad es relativamente adecuada con prestaciones mínimas y en proceso de estandarización. Los operadores estiman que el roaming es un servicio de alto potencial e intentan elevar el número de viajeros que lo emplean mediante la reducción de los cargos de terminación de los operadores locales y el tiempo aire (airtime charge), pero se enfrenta a la estrategia cortoplacista de los operadores sudamericanos que prefieren alto ingreso por cliente y bajo volumen de usuarios.

Los operadores de Sudamérica, mayormente consideran que el roaming es un servicio que deben brindar sus propios ingresos, paradójicamente no se realiza la inversión requerida para un buen servicio ni se implementa servicios de atención del cliente. Todos los operadores han migrado operaciones de la tecnología CDMA hacia la tecnología GSM que se caracteriza por su menor inversión por cliente, bajos costo de upgrade y servicio altamente normalizado. Los operadores se adecúan a las normas de operación de un organismo denominado GSMA que ha devenido en un regulador de facto cuyas decisiones, potencialmente, pueden colisionar con la normativa de los países. 
Brasil, al igual que México y USA, asigna una alta prioridad al servicio roaming. Sostenidamente, ha efectuado reuniones de trabajo para consolidar el servicio en la subregión, pero los resultados han sido pobres, debido al modelo vigente, que facilita la interconexión internacional a costo elevado, los que impide que el servicio inicie la esperada etapa de crecimiento exponencial semejante al modelo CPP (calling party pay) de mediado de los años noventa. El regulador ANATEL de Brasil considera que su desarrollo interno y su experiencia en itinerancia local, puede ser ampliada a los demás países, mediante el desarrollo de una infraestructura de roaming común, con costos bajos y sin intromisión de mayoristas privados internacionales,

\section{La visión de negocio de los operadores}

Las estimaciones sobre el mercado de roaming, indican que estos servicios pueden proporcionar hasta el $50 \%$ de los ingresos de las compañías celulares, especialmente en aquellos países que reciben $u$ originan mayor movilidad de personas, sea por turismo o por viajes de negocios.

El principal ejemplo de alto ingreso por el servicio se encuentra en el mercado europeo. Los operadores de los países la Unión con alto perfil turístico receptivo llegan al $40 \%$.

En América Latina, el servicio llegó hasta el 5\% en los operadores de alto ingreso de viajeros, pero en la mayoría se ha mantenido en niveles menores al $1 \%$, debido a los altos costos para el viajero y a la degradación de la capa de abonados postpago que, porcentualmente, representan un nivel bajo, dentro de la composición de clientes de los operadores de la región.

\section{La estrategia tarifaria}

La estrategia tarifaria se sustenta en cubrir costos del servicio, añadiendo un elevado margen de beneficio.

\section{El costo del servicio comprende:}

a. un componente fijo referido al acceso a la interconexión por los gateways, alquiler de enlaces, el manejo administrativo para la facturación e informática y la gestión administrativa de la operatividad y la post venta.

b. un componente variable para cubrir el tráfico medido en llamadas o minutos, el costo del intercambio de registros de facturación y la transmisión en línea de registros para control de alto uso indebido o fraude
La tarifa al cliente tiene un componente fijo y un componente variable. Esta tarifa, normalmente tiene un elevado margen, incluso se cargan montos superiores a los que se trasladan al cliente local por servicio de otros operadores (larga distancia).

La tarifa se conforma según el cuadro 4.

Cuadro 4. Valor de una llamada para el cliente

\begin{tabular}{|l|}
\hline Llamada saliente: \\
\hline $\begin{array}{l}\text { Tarifa al cliente }=\left(\mathrm{ci}+\mathrm{ar}^{*} \mathrm{~min}+\mathrm{dd}{ }^{*} \mathrm{~min}\right){ }^{*}(1+ \\
\text { taxes })\end{array}$ \\
\hline donde: \\
\hline ci $=$ cargo de interconexión \\
\hline ar $=$ airtime rate \\
\hline $\begin{array}{l}\text { Idr }=\text { long distance rate, transferido de operador } \\
\text { LD }\end{array}$ \\
\hline taxes = impuestos de ley \\
\hline Llamada entrante: \\
\hline Tarifa interoperadores $=($ ci + ar*min $) *(1+$ taxes $)$ \\
\hline donde: \\
\hline ci = cargo de interconexión \\
\hline ar = airtime rate \\
\hline taxes = impuestos de ley \\
\hline
\end{tabular}

Fuente: Acevedo, A. Ibid.

\section{LA ESTRUCTURACIÓN DEL SERVICIO}

\section{LA CALIDAD DEL ROAMING}

El servicio básico que el cliente viajero espera Todo viajero que emplea el roaming internacional desea acceder a un servicio activado y sencillo en el lugar visitado, con capacidad para efectuar y recibir llamadas de voz y, eventualmente, recibir tráfico de datos.

\section{La utilidad del servicio}

En roaming, lo que se espera es la movilidad internacional del número celular.

Esta movilidad debe cubrir los cuatro tipos de utilidad:

1) Utilidad de lugar. El servicio debe seguir y conectar al viajero. 
2) Utilidad de tiempo. El servicio debe estar siempre conectado y con la cobertura requerida.

3) Utilidad de uso. Debe ser útil y sencillo, al precio adecuado.

4) Utilidad de forma. Con equipos celulares amigables, de bajo riesgo para la salud del usuario.

\section{La calidad del servicio roaming}

La calidad del servicio se refiere a las expectativas de movilidad internacional de los viajeros.

Las variables del servicio son los siguientes:

a) La calidad de señal: potencia, continuidad, cobertura.

b) Los servicios suplementarios de voz: correo de voz, indetificador de llamante, transferencias.

c) Los servicios suplementarios de datos: SMS, MMS, navegador para acceso a datos.

d) Nivel de atención a los clientes y logro de fidelización.

No se realiza ninguna medición técnica de la calidad ya que no existe una norma de calidad del servicio roaming que considere las necesidades, expectativas y percepción del cliente.

\section{LA INFRAESTRUCTURA INTANGIBLE Y TANGI- BLE DEL SERVICIO}

\section{Premisas para el funcionamiento del servicio}

La creación de infraestructura para el funcionamiento del roaming se basa en varias premisas:

- Es una actividad de operadores de servicios, por lo que éstos lo brindarán e incentivarán si obtienen un rendimiento económico positivo.

- El roaming es independiente de la asignación de espectro, por lo que no es sometido a la normativa de los reguladores.

- Los operadores se conectan a través de proveedores intermediarios que cumplen la función de interconectar y traducir diferentes opciones técnicas, a fin de hacerlas compatibles.

- En roaming, existe el suficiente know how técnico, que permite la interconectividad de los operadores y la interoperabilidad entre diferentes versiones tecnológicas y entre diferentes tecnologías. Es posible el roaming entre un operador GSM y un operador CDMA a través de un intermediario (PARC, MACH SYNIVERSE) que realizarán las adecuaciones y traducciones que el servicio requiera.

- El tema crítico que afecta la interoperabilidad del roaming, es la compatibilidad tecnológica del ter- minal, el cual debe ser capaz de conectarse a la red visitada y soportar la interfaz aire del operador visitado.

- En el futuro cercano, la interoperabilidad roaming se referirá a la compatibilidad entre tecnologías de segunda y tercera generación $(2.5 \mathrm{G}, 3 \mathrm{G})$ y las futuras evoluciones de las tecnologías que se consoliden.

\section{Los segmentos de la infraestructura del servicio}

Hacia el interior de cada operador, el servicio roaming se estructura en tres segmentos claramente diferenciados: administrativo, técnico y equipo.

a) El segmento administrativo comprende el website, atención al cliente, facturación y control de tráfico indebido. El desarrollo, los acuerdos, la coordinación interoperadores e interfuncionales, la facturación retail (cliente) se manejan bajo administración propia y representan gastos administrativos.

b) El segmento técnico comprende la interconexión internacional, MSC, HLR, VLR, SMSC, MMC, LCF, clearinghouse, IOT. Se realiza inversión propia (switch) que representa costos y depreciación o se terceriza (interconexión y señalización internacional, clearinghouse, envío-recepción cdrs y formato de intercambio, IOT, antifraude) bajo modalidad de gastos de pago fijo o por volumen de tráfico.

c) El terminal o handset comprende interoperabilidad, multibanda y multifuncionalidad. Se adquieren y se transfieren para usufructo del cliente, como venta, alquiler o donación. Por fines prácticos no se considera gasto ni costo.

Hacia el exterior, el servicio es coordinado por un proveedor de servicios interoperadores, que permite que los segmentos técnicos de los operadores se interconecten y se interrelacionen en forma automática. La coordinación del segmento administrativo no se encuentra estructurada y se sujeta a las coordinaciones personales e intereses de los responsables (ver la figura 2).

\section{Los niveles para el manejo del roaming en la em- presa}

El roaming internacional es un servicio de funcionamiento sencillo, pero la multiplicidad de variables intervinientes en operatividad, ha determinado que su organización administrativa y de decisiones haya sido soslayada y postergada.

Se han desarrollado dos tipos de perspectiva en los operadores. Por un lado, la priorización de la movi- 
Figura 2. Elementos básicos para la creación de infraestructura en roaming internacional

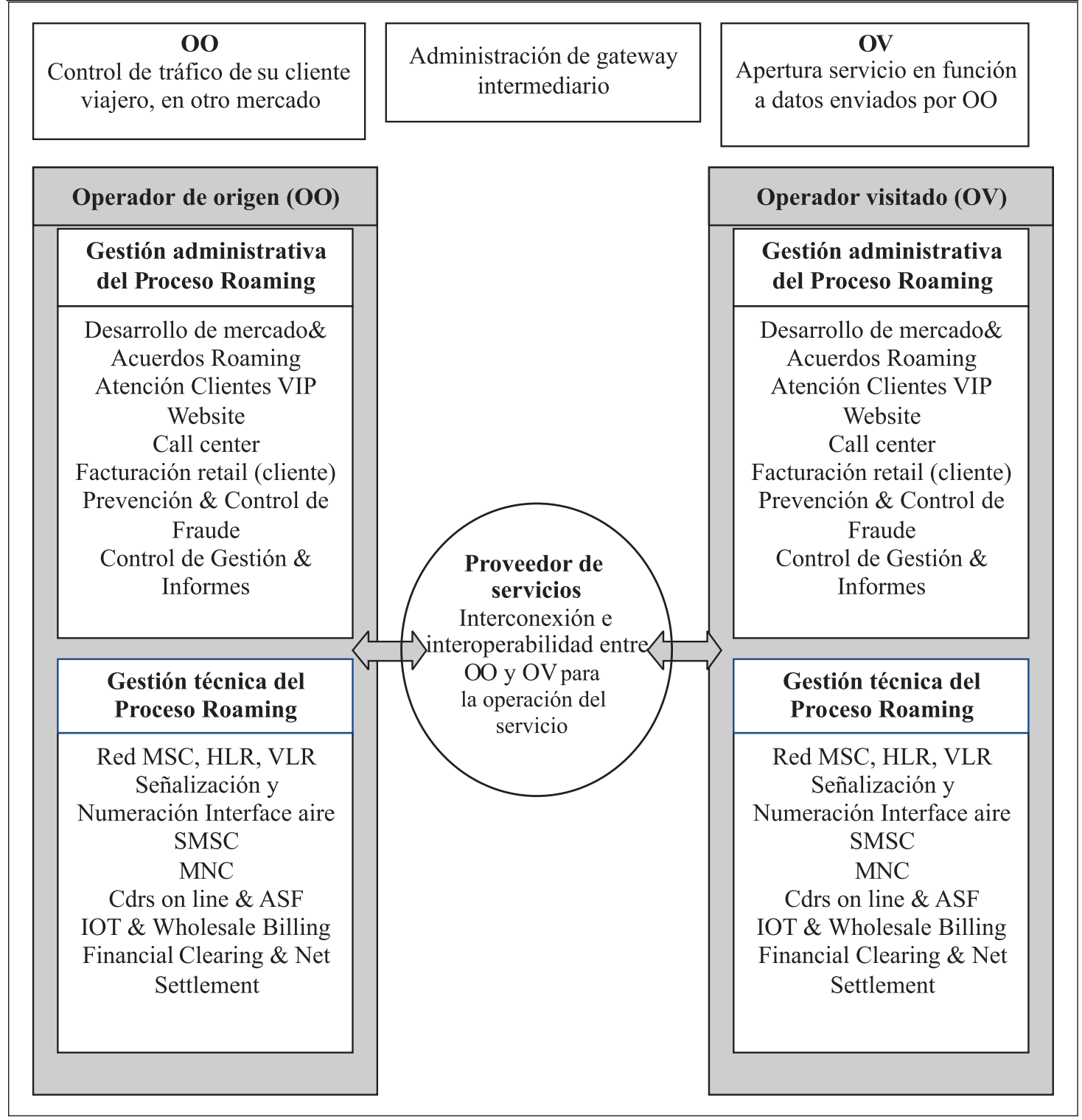

Gestión técnica: MSC (mobile switching center), HLR (home location register), VLR (visitor location register), SMSC (short message service center), MNC (mobile network code), Cdr on line (call detail record on line), ASF (antifraud system force), IOT (inter operator tariffs), Wholesale Billing (facturación mayorista), Financial Clearing (intercambio financiero), Net Settlement (liquidación neta).

Fuente: Elaboración propia.

lidad internacional de los clientes y la asignación de mayores recursos para la consolidación del área roaming. Por otro lado, la reducción del área y mimetización de sus atribuciones dentro de las tareas normales de las áreas funcionales, bajo la consideración que la nueva tecnología trae operatividad sencilla, sin riesgos y normas mercerizadas y conocidas.

Acerca de la departamentalización, cada compañía ha diseñado su propio arreglo organizativo. Todo operador posee un área roaming que ubica dentro de cualquier área funcional de la empresa, en la perspectiva que donde se encuentre, siempre funcionará igual y casi sin intervención gerencial.

El operador exitoso maneja el servicio con una arquitectura multidimensional, que supera las estructuras clásicas de coordinación matricial. Considera el manejo a tres niveles:

- A nivel táctico-operativo el roaming se organiza como una unidad no jerárquica que utiliza el enfoque de proceso para coordinar los puntos de 
actividad de roaming a cargo de las áreas especializadas. Los puntos de actividad roaming se ubican en las gerencias funcionales y corresponden a una porción de las actividades normales de dichas áreas. El área de roaming coordina y alinea los recursos locales con los recursos de otros operadores y proveedores, para la señalización, el STP-gateway, control high usage, uso e intercambio de registros de tarificación (CDRS) y facturación entre los operadores.

- A nivel estratégico-directivo, el roaming se maneja como un proceso de negociación y toma de decisiones sustentada en el flujo de información de uso (tráfico) y financiero (neteo, cobros y pagos), que equilibran el tráfico del servicio y la mayor rentabilidad con el riesgo de uso indebido y las pérdidas por no facturables. Se encarga del diseño e implantación de proyectos relacionados con la provisión del servicio, tales como la red de señalización, Signaling Transfering Point, Punto de Recepción autorizado, facturación y neteo centralizado, ARP-clearinghouse, Control de tráfico. Posee un alto componente de interrelaciones personales para mantener y mejorar la operatividad y para negociar y resolver los problemas operativos, administrativos, financieros o legales que surgen.

- A nivel conceptual se considera el concepto de producto que deriva en el diseño de producto, desarrollo y operación, incluye cadena de servicio, identificación de restricciones y cuellos de botella operativos, administrativos, financieros 0 legales, y los mecanismos que equilibren inversión y costo operativo. Las decisiones comprenden la visión del servicio, la orientación de la migración tecnológica, la integración operativa con la perspectiva del largo plazo.

\section{LA VISIÓN ACERCA DEL FUTURO DEL ROA- MING}

\section{LOS MODELOS DE CRECIMIENTO DE ROAMING}

\section{Los modelos de voz en roaming}

En el camino de consolidar el roaming en América Latina se han desarrollado diferentes modelos, aplicados en las diferentes etapas del ciclo de vida del servicio.

El primero modelo se denomina acuerdo bilateral donde cada operador establece acuerdo roaming individual con otro operador cuyo mercado le es atra- yente. Este modelo estuvo vigente en la etapa incipiente del roaming, y presentaba el inconveniente de generar un voluminoso esfuerzo administrativo, bajos ingresos, elevado fraude y servicio deficitario.

En el segundo modelo se realizan acuerdos bilaterales y se interconectan a través de un gateway, que centraliza la red de interconexión y la señalización, a través de la cual cada operador obtiene acceso a los mercados donde posee acuerdos roaming vigentes. La tarea administrativa se aligera con el intercambio de registros de tarificación (CDRs) y el neteo de liquidaciones a través de un clearing. Es el modelo actual vigente en tecnologías $2 G$ y $2.5 G$.

El tercer modelo es una sofisticación del segundo y se denomina hub roaming, donde un gateway brinda acceso a las redes y controla la facturación y el fraude. El hub es un piggy back ampliado que busca alto rendimiento, rápida ampliación de la cobertura y bajos gastos administrativos. Mediante una única conexión de red, los operadores acceden al servicio, además de intercambio de datos, neteo y liquidación financiera y protección contra el fraude.

\section{El nuevo modelo de datos en roaming}

Con el avance de la convergencia y la banda ancha de alta velocidad, se está estructurando el modelo de roaming IP, donde se utiliza un gateway IP como puerta de entrada a un operador de red Internet que permite acceso a la red y transmisión (tx) de datos cuando se encuentra en roaming. Esto se muestra en la figura 3.

Se estima que, en el futuro, este modelo basado en Internet será la base para un roaming superior de voz a través de protocolo Internet.

Pero esta posibilidad, no es atrayente para los operadores actuales, ya que el modelo de negocio vigente propicia la exclusión de los clientes normales, la concentración en un segmento insensible al precio, y el alto rendimiento basado en tarifas elevadas. Con bajo crecimiento.

El futuro roaming IP requiere el diseño e implantación de un adecuado modelo de negocio, el que, aún, es inexistente.

\section{ELEMENTOS PARA EL NUEVO MODELO DE NE- GOCIO}

Se destaca que, en la región, se han dado casos exitosos que permitieron el crecimiento del servicio. Por ejemplo, ENTEL PCS fue el primer operador 
que migró de tecnología en la región, su decisión de implantar la primera red GSM del continente fue determinante en el largo plazo. AT\&TW proporcionó sus puntos de código para la implantación de RAI, de no hacerlo, el servicio no hubiera sido posible. El grupo roaming de Telefónica que implantó su propia red de roaming, la que fue desmantelada posteriormente.

Los temas de decisión clave, que brindarán las bases para el desarrollo y consolidación del servicio roaming, en sus componentes de voz y de datos, han de ser los siguientes:

\section{Política de los gobiernos}

- Encontrar el enlace entre los objetivos de reducción de la exclusión social y económica, el impulso de las tecnologías de información, accesibilidad a roaming y sectores emergentes.

- Definir los aspectos que intervención de los gobiernos en roaming, para incentivar el servicio.

- Cómo integrar las políticas públicas, considerando su consolidación regional en los temas de TIC, Internet Protocol, roaming.

- Definir y enfrentar el abuso de posición dominante en roaming internacional.

- La creación de mercado en roaming, a partir de la ola de reformas en la normativa.

\section{Normativa de roaming}

- Determinar si la normativa en roaming es necesaria, considerando la estructura industrial y la tendencia a la madurez del sector.

- Enfocar la normatividad en el roaming.

- Posición frente al papel normativo que la GSMA ha asumido, intentando reemplazar a la regulación de los países.

- Adoptar los nuevos mecanismos de control de tarifa en roaming, como en Europa.

\section{Desarrollo del mercado}

- El mercado de telefonía celular es concentrado en dos o tres operadores por país que conforman bloques regionales. La cuestión es determinar si es posible la creación de condiciones de competencia y/o competitivas.

- Considerando que el servicio móvil está llegando a la madurez (100\% celulares/población en Europa y Asia, más del $50 \%$ en América Latina), se deben plantear las acciones para que roaming se enfoque en el crecimiento.

- Tecnológicamente existe la oferta de servicio de roaming para todos los usuarios, contrato o pre- pago. En la realidad, es administrativamente complejo, el intento de ampliar servicio hacia prepago.

- En la búsqueda de la integración regional, se debe promover un acuerdo regional para la creación de un organismo supranacional que integre los esfuerzos locales, para desarrollo del servicio.

- Se debe diseñar el modelo de negocio, considerando el potencial de rendimiento económico de los servicios.

\section{Inversión en infraestructura}

- El roaming es un elemento que conforma la renovación tecnológica de los operadores, de manera que es un costo hundido que no está siendo aprovechado.

- Con las nuevas versiones tecnológicas que facilitan la ubicación extraregional de la infraestructura física, se debe verificar y controlar la infraestructura virtual o asentada en otros países.

- Se debe manejar el aumento del riesgo de fraude derivado de los nuevos dispositivos con tecnologías de tercera generación.

- Estudiar las maneras para estimular las inversiones en roaming.

\section{ELEMENTOS PARA EL DISEÑO DE LA ARQUI- TECTURA DEL ROAMING}

Para crear un servicio rentable, en crecimiento e inclusivo de todos los segmentos de la organización, se necesita un nuevo enfoque conceptual que sustente las bases para diseñar nuevos escenarios futuros y para desarrollar visiones holísticas del sector y del servicio.

Desde la perspectiva de la dirección estratégica y sociotécnica (Acevedo, 2003), el roaming, debe considerar el objetivo regulatorio de mejorar las condiciones de acceso a la población, el objetivo empresarial de crear las condiciones para la consolidación del negocio rentable y el objetivo de marketing orientado a satisfacer las necesidades de todos los segmentos de usuarios.

A partir de la identificación de la oportunidad de negocio que representa el servicio roaming internacional, se muestran las dimensiones del sector telecomunicaciones y del negocio celular relacionados al servicio de roaming internacional (figura 4).

\section{En la dimensión estructural,}

La UIT y el GSR-08 (2008) buscan el fomento de la inversión en el sector, para lo cual, están promo- 
viendo la reforma reglamentaria a nivel mundial, que ha de considerar los siguientes aspectos:

- Sobre redefinición de la normativa internacional:

* Roles y funciones de los actores (gobierno, reguladores, operadores, mayoristas, minoristas) separados y diferenciados.

* Nuevas reglamentaciones, derivadas del surgimiento de tecnologías emergentes.

* Previsión de las normas sobre la convergencia e innovación de servicios.

- Sobre redes e infraestructura de comunicación:

* Acceso libre a las redes de cable submarino.

* Liberalización de las pasarelas internacionales.

* Compartición de la infraestructura, para que los usuarios excluídos puedan acceder a los servicios.

- Sobre desarrollo de inversión en infraestructura para roaming:

* Definición de las reglas (por primera vez) para funcionamiento de roaming entre países.

* Tipo de infraestructura básica que requiere cada país y operador.

* Definición del nivel atribuciones y alcance de la regulación local frente a la cobertura internacional de roaming.

\section{En la dimensión de proceso:}

La globalización requiere la movilidad plena de las personas, equipos de comunicación y los números de telefonía para contactar a las personas. Se están diseñando las estrategias y los pasos a seguir para crear un servicio de roaming internacional de bajo precio y de altas prestaciones.

Según los criterios de ITU (2008), se consideran los siguientes aspectos:

- Temas del modelo de negocio:

* Competencia-mercado.

* Operadores virtuales MVNO.

* Socio global/socios globales.

* Alianza con el operador clave.

* Inversión en el negocio: inversión en infraestructura.

* El adecuado Modelo de negocio: datos, roaming.

* Acuerdos de itinerancia.

* Ventaja competitiva y Cadena de valor.

* Ingresos /Utilidades. Margen de contribución por negocio.

- Estrategia Legal-regulatoria de roaming:

* Secuencia de intervención regulatoria en roaming.
- Tarifa roaming retail (al cliente).

- Atención al cliente: y defensa de usuario.

- Acuerdo regional y por países.

- Permisos locales y acceso a redes de terceros

- Costos por servicio y condiciones de facturación

- Delimitación de los acuerdos interoperadores.

* La Regulación en roaming por la Unión Europea.

- Control IOT(inter operador tariffs) en roaming internacional.

- Control de tope tarifario en roaming.

- Fijación de la tarifa al cliente.

\section{En la dimensión de persona}

La exclusión económica y social de los grupos marginales (rurales y urbano-marginales) se encuentra en nivel crítico en los países atrasados.

Los temas a considerar son:

- Desarrollo de la sociedad de la información:

- Objetivos de acceso e inclusión.

- Acceso universal.

- Servicio universal.

- Metas de reducción de la brecha digital.

- Proyectos de e-educación y ciudades digitales.

- Desarrollo de la capacidad de decisión de los usuarios:

- Libertad de elegir por el usuario.

- Competencia real en el sector.

- Diversas opciones, a los costos adecuados.

- Segmentación de mercado para ofrecer servicio a diferente costo y tarifa.

\section{En la dimensión del cambio}

El cambio en el sector se sustenta en el modelo de innovación vigente (Linares 2005) y la nueva estructura que está tomando la economía en red (Kelly 1998) y la tendencia del desarrollo tecnológico que viene siendo orientado por la ITU y Citel (UMTS 2007).

Los temas a considerar son:

- Cambio en el entorno:

- Tendencias tecnológicas y sociales.

- Riesgos y escenarios del sector

- Dinámica de la innovación tecnológica.

- Ciclo de vida de los servicios

- Cambios en la tecnología:

- Ciclo económico de tecnologías

- Convergencia de sectores de comunicación, computación, entretenimiento e información. 


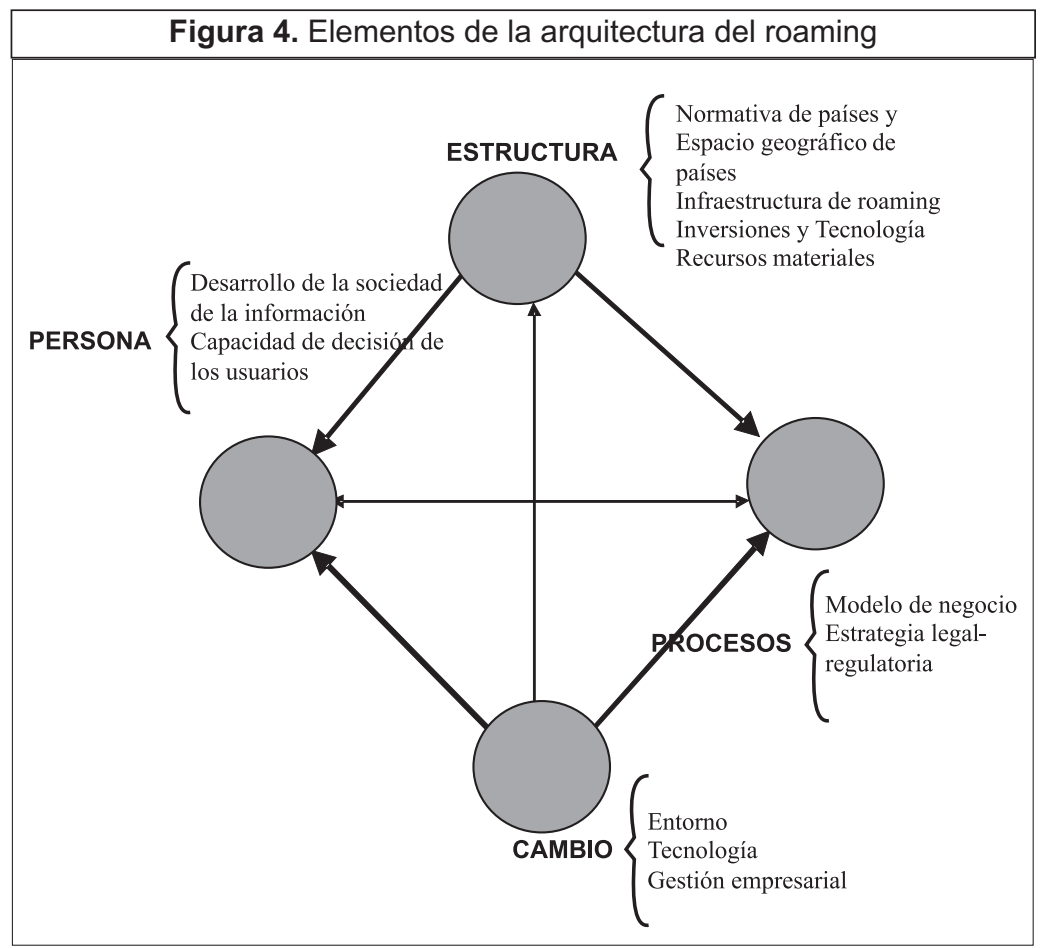

Fuente: Elaboración propia

- Incertidumbre del éxito comercial de las nuevas tecnologías.

- Proyectos y planes de desarrollo. Cronogramas de avance a Corto plazo e hitos.

- Cambios en la gestión empresarial:

- Benchmarking entre operadores y adopción de las mejores prácticas.

- Aplicaciones innovadoras en países emergentes.

- TIC para el desarrollo.

\section{CONCLUSIONES}

En el presente estudio, se ha ensayado una interpretación para explicar las debilidades estructurales y la pobre dinámica de crecimiento del servicio roaming, definido como un producto ofrecido por los operadores de telefonía móvil y considerado el de mayor potencial en la década pasada.

Con la estrategia de alianzas mundiales y de ampliación de mercados con acuerdos de libre comercio, el servicio roaming adquiere especial relevancia. La inversión en infraestructura significaría la consolidación del servicio, clave para la comunicación e integración de las alianzas empresariales, por lo que, su desarrollo está considerado dentro del proyectos de creación de infraestructura del BID.
En la dimensión del devenir, se describen las razones y la manera en que se dio inicio y se desarrolló el roaming internacional entre operadores de América, el cual tomó un nuevo cariz al momento de las privatizaciones, que trajeron cambios tecnológicos, redefinieron la organización industrial de telecomunicaciones y ampliaron las misiones y objetivos empresariales.

En la dimensión del proceso, se define el negocio roaming, enmarcado dentro del avance tecnológico que es la oferta concreta de producto. Este negocio se ha implantado a partir de las estrategias de desarrollo de servicio y la segmentación y captación de clientes. Se ha operacionalizado a través de la política de precio basado en valor y no en costo y la concentración en la satisfacción de un reducido número de usuarios.

En la dimensión estructural, se definen las variables de calidad que debe cubrir el roaming, algunos elementos de la calidad técnica del servicio, la organización del roaming en sus segmentos técnico y administrativo y la organización internacional basado en un modelo de arquitectura multidimensional, actualmente se ha reducido su nivel $y$ capacidad coordinadora, limitando su alcance a ser intermediario operativo entre las unidades funcionales de ventas, atención postventa, facturación o de gestión de redes. 
En la dimensión humana, se ha mostrado una visión conceptual acerca de lo que se requiere para rediseñar un adecuado modelo de negocio que facilite el crecimiento del servicio, paralelo al del crecimiento de los negocios de telecomunicaciones. Debe complementarse las visiones de los actores que intervienen en el sector: legisladores, ejecutivos de la industria, managers de los operadores, consultores y asociaciones.

Para crear un servicio rentable, en crecimiento e inclusivo de todos los segmentos que lo requieren, se necesita un nuevo enfoque conceptual que siente las bases para diseñar nuevos escenarios futuros y para desarrollar visiones holísticas del sector y del servicio.

Este nuevo enfoque ha de brindar renovadas perspectivas que permitan ver y entender los modelos mentales vigentes en el sector. Entender y superar aquellos modelos mentales que demostraron su eficacia en la fase de crecimiento pero que se han convertido en rémoras mentales que ocultan las externalidades negativas del sector, impiden el desarrollo equilibrado y retrasan que el sector cumpla con su misión de acceso universal y de inclusión.

\section{REFERENCIAS}

[1] Acevedo, Adolfo (2008), "Calidad e interoperabilidad en el servicio roaming: claves para el modelo de negocio". En www.iirsa.org/BancoEvento/T/taller_iirsa_citel/ (página visitada 20 abril del 2008).

[2] Acevedo, Adolfo (2003), "El modelo de los sistemas sociotécnicos en la teoría y praxis empresarial", USIL-CLADEA. Lima-Perú.
[3] Band, William (1991), Creating Value for Customers, Moving From Talk to Action, John Willey \& Sons, Inc. New York.

[4] CDG (2004), CDMAAmericas Congress 2004. En http://www.ebizlatam.com/ newsletter/ pdfs (página visitada 20 noviembre del 2005).

[5] Citel OEA. Documento Coordinado de Normas N. ${ }^{\circ}$ 1. COMUNICACIONES INALÁMBRICAS, en http://www.umtsforum.net/ (visitado 10 de febrero del 2008). En:

http://www.3gamericas.org/ (página visitada 11 de febrero del 2008

[6] GSM Association (2006), PRD BA.40, v4.1, Roaming Guide. UK.

[7] ITU (2008), Simposio Mundial para Organismos Reguladores (GSR-08), Pattaya, Tailandia. En:

http://www.itu.int/newsroom/features/indexes.html (página visitada 30 de marzo del 2008).

[8] Kelly, Kevin (1998), Nuevas reglas para la nueva economía, Ediciones Granica S.A. México.

[9] Linares, Carolina (2005), "Nota Técnica sobre innovación tecnológica en empresas de servicios", Universidad de Chile, Santiago de Chile.

[10] Regulatel-Ahciet (2007). "Armonización y Universalización". X Cumbre de Reguladores y Operadores. Madrid, España. En www.ahciet.net/Eventos (página visitada 01 de marzo del 2008).

[11] UMTS (2007), A view of the future. Spectrum for future development of IMT-2000. En www.umtsforum.net/ (página visitada 22 enero del 2008).

[12] Universidad del Cauca (2003), "ROAMING 3G/WLAN - La Internet Móvil e Inalámbrica "Permanente", en Revista Enlace Infomático. 